\title{
THE ESTIMATION OF PIG BONE AGE FOR FORENSIC APPLICATION USING THERMOGRAVIMETRIC ANALYSIS
}

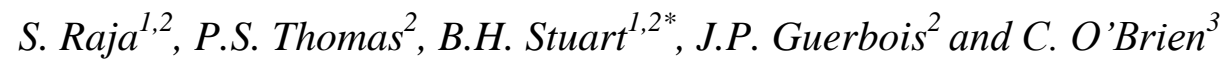

${ }^{1}$ Centre for Forensic Science, University of Technology, Sydney, PO Box 123, Broadway NSW 2007, Australia

${ }^{2}$ Department of Chemistry and Forensic Sciences, University of Technology, Sydney, PO Box 123, Broadway NSW 2007, Australia

${ }^{3}$ University of Ontario Institute of Technology, Faculty of Science, 2000 Simcoe St North, Oshawa ON L1H 7K4, Canada

* Corresponding author

\begin{abstract}
An accurate means of determining bone age is a goal for forensic scientists. In this study, thermogravimetric analysis (TGA) has been used to examine pig bone specimens of different post-mortem age. Analysis of bone in both air and nitrogen atmospheres reveals a decrease in total mass loss as the bones age. Two mass loss steps due to the decomposition of the organic bone components were observed and show decreasing trends with age for decomposition in an air atmosphere. In a nitrogen atmosphere the decomposition was observed to be more complex and age dependence of the mass loss for each step was not identified. The TGA data, however, demonstrates the potential of the technique as a means of estimating postmortem age of forensic bone specimens.
\end{abstract}

Keywords: bone; decomposition; forensic; thermogravimetric analysis. 


\section{Introduction}

Gaining an understanding of the ageing of bone fragments or decomposed skeletal remains provides a challenge for forensic practitioners. Bones are complex in structure and are sensitive to environmental factors. Although extensive studies of the visual and physical properties of bones have been carried out, there have been fewer investigations into the changes to the chemical structure of bones in a forensic context. As bones contain both inorganic and organic components, there is potential to gain an insight into the post-mortem decomposition processes occurring using techniques that are sensitive to changes to such components.

Thermal methods lend themselves to the examination of both the inorganic and organic components of bone. The use of thermal analysis to the study of bone decomposition has been previously reported [1-9]. Thermogravimetric analysis (TGA) has been employed in a number of studies to the characterisation of bones. TGA data shows mass losses at particular temperatures that may be correlated with the organic and inorganic phases present in bone. A step in the temperature range $50-220^{\circ} \mathrm{C}$ is associated with water loss, while a second step appearing up to $600^{\circ} \mathrm{C}$ represents the decomposition of organic components. A third step in the range $650-850^{\circ} \mathrm{C}$ is associated with the release of carbon dioxide from the carbonated hydroxyapatite if the decomposition is carried out in an air atmosphere, but is also associated with the further decomposition of the products of pyrolysis produced in the second step if the decomposition is carried out in an inert atmosphere [6]. TGA has been previously used to characterise fresh bone [5], to examine the effect of heat treatment on bone [7] and for the dating of post-mortem bones [9]. In the latter study, bones ranging in post-mortem age from 6 months to 40 years, with most specimens of greater than age 10 years, were investigated. 
Although differences between young and old bones are detectable, there is an interest in determining differences between younger bones for forensic purposes. In the current study, TGA has been carried out to characterise bone specimens obtained from pig bones of young post-mortem age exposed to identical burial conditions.

\section{Materials and methods}

Bone samples

Bone specimens with post-mortem ages ranging between 3 months and 7 years were obtained. All samples were flat rib bones from female pigs obtained from the same farm with an identical diet and weighing 40-45 kg. The pigs were buried in soil $60 \mathrm{~cm}$ below the surface for a designated amount of time. The average ambient temperature was $25^{\circ} \mathrm{C}$ and the soil was slightly acidic. After exhumation the bones were stored in sealed in plastic bags at $4^{\circ} \mathrm{C}$ prior to analysis.

The compact bone specimens were cleaned only by scraping of the surface with a scalpel to remove fatty bone marrow from the interior and any residues from the exterior of the specimens [6]. The bones were mechanically sliced using a Buehler IsoMet low speed diamond saw into $2 \mathrm{~mm}$ thickness slices. Each sample was dried in a vacuum oven at approximately $50^{\circ} \mathrm{C}$ for $2.5 \mathrm{~h}$ to remove moisture. The slices were then cut in half using a scalpel.

\section{Thermogravimetric analysis}

A TA Instruments SDT 2960 was used to analyse the bone samples. Samples (14-17 $\mathrm{mg}$ ) were placed in a platinum crucible and an empty platinum crucible was used as a reference. Two atmospheres were used for each sample type: air and $\mathrm{N}_{2}$. The flow rate of air 
was $20 \mathrm{~mL} \mathrm{~min}^{-1}$ and the rate of $\mathrm{N}_{2}$ was $150 \mathrm{~mL} \mathrm{~min}^{-1}$. Samples were heated from ambient temperature up to $1000^{\circ} \mathrm{C}$ at a temperature gradient of $10^{\circ} \mathrm{C} \min ^{-1}$.

Due to the potential variability in the water content of the bone specimens, the TG data is presented as a function of the final mass $\left(\mathrm{m}_{\mathrm{final}}\right)$. TG curves in Figures 1 and 2 are calculated as a percentage of the final mass resulting in an apparent 'mass gain' (i.e. $\left.\left(\Delta \mathrm{m}+\mathrm{m}_{\text {final }}\right) / \Delta \mathrm{m}_{\text {final }}\right)$. Mass loss data presented in Figures 3 to 6 are calculated as a percentage of the accumulated mass to the final mass (i.e. $\left.\Delta \mathrm{m}_{\text {step }} /\left(\Delta \mathrm{m}_{\text {step }}+\mathrm{m}_{\text {final }}\right)\right)$.

\section{Results and discussion}

Figure 1 illustrates the TG curves obtained for a specimen of bone with a 3 month post mortem age in both air and $\mathrm{N}_{2}$ atmospheres. The data has been plotted as a function of '\% mass gain' based on the final mass rather than the more traditional mass loss as water content can be variable resulting in significant variation in percentage mass losses based on initial mass. It is typical also to plot the data as the derivative of mass loss (DTG) to help identify the number of decomposition processes (Figure 2). For the 3 month sample shown in Figure 2, for both the air and $\mathrm{N}_{2}$ atmospheres, four mass loss steps are observed. A water loss step is observed below $220^{\circ} \mathrm{C}$. There are two steps associated with the thermal decomposition of organic matter at $220-420^{\circ} \mathrm{C}$ and $420-600^{\circ} \mathrm{C}$. The first stage of organic decomposition involves the breakdown of polymeric material into solid and volatile degraded substances and the second stage involves the further breakdown of the solid degraded substances. A small step, step 3 is observed above $600^{\circ} \mathrm{C}$ and is associated with carbonate decomposition in an air atmosphere, but also includes further pyrolysis of the organic matter in the inert $\left(\mathrm{N}_{2}\right)$ atmosphere. The mass loss steps are fairly consistent for the decomposition of the air atmosphere samples up to 60 months post-mortem age. The pyrolysis in an inert atmosphere, 
however, was observed to be more complex resulting in an increase in the number of mass loss steps observed.

The trends in total mass loss for both atmospheres are illustrated in Figures 3 and 4. Figure 3 illustrates the total mass loss based on the accumulated mass loss to $1000^{\circ} \mathrm{C}$ (i.e. excluding the water component) in an air atmosphere as a function of bone age. The $\%$ mass loss decreases with age, indicating structural change as the bone ages. Figure 4 shows the mass losses determined for bones examined in a $\mathrm{N}_{2}$ atmosphere. In both cases the total mass loss (step $1+\operatorname{step} 2+$ step 3$)$ is seen to decrease with increasing age up to approximately 2 years of ageing (23 months). Above this age mass loss does not significantly change. There is a reasonable amount of scatter in the data (deviation of up to 4.9 months from the linear fit with an average deviation of 2.7 months for the air atmosphere decomposition and deviation up to 6.3 months with an average deviation of 3.7 months for the $\mathrm{N}_{2}$ atmosphere decomposition). It is clear, however, that there is a relationship between the total mass loss in both atmospheres and the post-mortem age of the bones up to 2 years of age.

The decomposition in the nitrogen atmosphere was observed to be complex with multiple peaks being developed with increasing age. The air samples, however, showed a consistent two step mechanism of the oxidative decomposition of the organic matter in the bone specimens. Deconvolution of steps 1 and 2 in Figure 2 in the air atmosphere data was carried out using both the DTG data and the second derivative DDTG data to identify the mid point of the two step process. Mass loss data for the two steps are shown in Figures 5 and 6 for steps 1 and 2, respectively. An improved correlation between post-mortem age and step 1 mass loss is observed with a maximum deviation from the linear fit of 4.0 months with an average deviation of 2.3 months. Step 2 also shows improvement in the correlation up to 23 months with a maximum deviation from the linear fit of 4.5 months and an average deviation 
of 1.9 months. Increased scatter in the step 2 data above 23 months, however, reduce the strength of this correlation.

The application of TGA to the determination of post-mortem age of bones specimens has been show to be possible in longer time frames (10 to 40 years) [9], however, little data has been published to demonstrate the potential of this technique for the determination of post-mortem age for much shorter time periods. The data acquired in this investigation suggests that TGA has the potential to be used in this regard. The preliminary data included in this paper shows some variability in the correlation between mass loss and post-mortem age. However, a relationship has been shown to exist between mass loss and post-mortem age of less than 24 months giving TGA the potential to be part of the armoury for the ageing of bone specimens.

\section{Conclusions}

TGA analysis of bones of different post-mortem age reveals that the total mass loss decreases with age up to 23 months of ageing. In both air and $\mathrm{N}_{2}$ atmospheres, the DTG curves show two main steps in mass loss, with both steps representing the loss of the organic component of bones. There is a decreasing trend of mass loss with age at each step. The results of these studies demonstrate the potential of TGA as a technique for estimating the post-mortem age of forensic bone specimens as the mass loss due to the organic component of bone is sensitive to age.

\section{Acknowledgements}

The authors would like to thank the Centre for Forensic Science, University of Western Australia, for assistance with this study. 


\section{References}

1. G.E. Fantner, H. Bikedal, J.H. Kindt, T. Hassenkam, J.C. Weaver, J.A. Cutroni, B.L.

Bosma, L. Bawazer, M.M. Finch, G.A.G. Cidade, D.E. Morse, G.D. Stucky and P.K. Hansma PK, Bone 35 (2004) 1013.

2. Y. Okamoto, S. Hidaka, Y. Yamada, K. Ouchi, K. Miyazaki and S.Y. Liu, J. Biomed. Mater. Res. 41 (1998) 221.

3. M. Utech, D. Vuono, P. De Luca and A. Nastro, J. Therm. Anal. Cal. 80 (2005) 435.

4. L.D. Mkukuma, J.M.S. Skakle, I.R. Gibson, C.T. Imrie, R.M. Aspden and D.W.L. Hukins, Calc. Tiss. Inter. 75 (2004) 321.

5. F. Peters, K. Schwarz K and M. Epple, Thermochim. Acta 361 (2000) 131.

6. A. Onishi, P.S. Thomas, B.H. Stuart, J.P. Guerbois and S. Forbes S, J. Therm. Anal. Cal. 88 (2007) 405.

7. C.Y. Ooi, M. Hamdi and S. Ramesh, Ceramics Int. 33 (2007) 1171.

8. A. Onishi, P.S. Thomas, B.H. Stuart, J.P. Guerbois and S.L. Forbes, J. Therm. Anal. Cal. $92(2008) 87$.

9. E. Villanueva, F. Girela and M. Castellanos, J. Forensic Sci. 21 (1976) 822. 


\section{Figure legends}

Figure 1. TGA curves for a bone specimen ( 3 months post-mortem) decomposed in air and $\mathrm{N}_{2}$ atmospheres. The data is represented as 'mass gain' based on the final mass.

Figure 2. DTG curves for the bone specimen decomposed in Figure 1 showing the four mass loss steps of thermal decomposition.

Figure 3. Mass loss (above $220^{\circ} \mathrm{C}$ ) versus bone age in an air atmosphere.

Figure 4. Mass loss (above $220^{\circ} \mathrm{C}$ ) versus bone age in a $\mathrm{N}_{2}$ atmosphere.

Figure 5. Mass loss data for step 1 for samples decomposed in an air atmosphere.

Figure 6. Mass loss data for step 2 for samples decomposed in an air atmosphere. 